\title{
Monotone Profitability Ranking in a Differentiated Oligopoly
}

\author{
Bing Jing \\ 44 West 4th Street, KMC 8-80 \\ Stern School of Business, New York University \\ New York, NY 10012 \\ U.S.A. \\ bjing@stern.nyu.edu
}

September 2, 2004

\begin{abstract}
In a setting where firms produce goods of distinct qualities at potentially different unit costs, I identify sufficient conditions for producing a higher quality good to be more (less) profitable.
\end{abstract}

Keywords: Vertical Differentiation, Bertrand Oligopoly, Imperfect Competition.

JEL Classification: D43, L13. 


\section{Introduction}

In this paper I consider a market where a fixed number of firms each offer a product of distinct quality. Production has constant returns to scale, although the firms may have different unit costs. Both the product qualities and unit costs of the firms are fixed exogenously, and they compete through setting prices simultaneously. Consumers have multiplicative utility functions that are increasing in quality, but they differ in their willingness to pay for quality. In such a standard setup of a quality-differentiated industry, the central question I investigate is the following: When is a firm producing a higher quality product more (less) profitable at equilibrium? Or equivalently, under what cost-quality conditions in the industry will offering a higher-quality product imply a competitive (dis)advantage? I consider both profit and profit margin as alternative indicators of profitability. Profit margin is essentially a firm's choice variable due to its one-to-one relationship to price. Profit margin is of independent interest also because it reflects the profit made from each unit sold.

In the familiar setting described above, at equilibrium a higher-quality product must be offered at a higher price (otherwise those products of lower qualities would not be purchased by any consumer), and products of higher qualities appeal to consumer segments with higher willingness to pay. If all firms were to produce at the same level of unit costs, therefore, a firm selling a higher quality product would naturally have a competitive advantage and enjoy greater profitability. However, the picture becomes much less clear when the firms have heterogeneous unit costs, because the profitability of each firm depends on not only its own cost-quality profile but also those of the other firms. A major contribution of this paper is to develop two measures that each index a firm's productive competency, and identify the corresponding sufficient conditions for producing a higher quality product to be more (or less) profitable.

The first measure is a firm's absolute cost efficiency, defined as the ratio 
between its quality level and unit cost. It is an absolute measure in that it is determined purely with each firm's own inherent characteristics. I show that a firm obtains both higher profit and profit margin if it can produce a higher quality product with an equal or higher absolute cost efficiency. This implies that, if the absolute cost efficiency is non-decreasing in quality, profitability is monotone increasing in quality. However, this measure can not predict the firms' profitability when the absolute cost efficiency is decreasing.

To address the deficiency of the previous measure, I develop a second metric which I call the relative cost efficiency of a firm. Unlike absolute cost efficiency, a firm's relative cost efficiency takes into account the costs and product qualities of both the firm itself and its immediate competitor(s). I show that, when the relative cost efficiency increases (decreases) in the quality levels of the firms, selling a higher-quality product is more (less) profitable. The cost trajectories that yield monotone increasing profitability can be concave, convex, or inversed-S-shaped (i.e., first concave and then convex as quality increases,) and the cost trajectories that yield monotone decreasing profitability can be convex or S-shaped (i.e., first convex and then concave as quality increases.)

The above results are obtained based on the assumption that the distribution of consumer types has a logconcave density. This paper also considers the special case of a uniform consumer distribution, and arrives at further results. The lower and upper bounds on each firm's profit margin are established. When the cost trajectory is (locally) concave over some quality range, the profit of a firm whose product quality is in the interior of this range can not exceed that of (at least) one of the two firms at the boundary of this quality range.

In the extant literature, two papers related to the present paper are Shaked and Sutton (1982) and Gal-Or (1985). In a duopolistic setting, Shaked and Sutton (1982) focus on demonstrating the existence of and also characterizing the (sub-game) perfect equilibria in qualities and prices. One 
result of theirs is that the firm choosing a higher quality level makes a higher profit. Gal-Or (1985) focuses on the impacts of entry on the degree of product differentiation and the average quality in the industry. Her model is one of Cournot competition, where a number of firms compete by choosing qualities and quantities to produce simultaneously. She derives a similar result that a firm choosing a higher quality level also obtains a higher profit. There are two major differences between these two papers and the current paper. First, their models assume specific forms of unit cost functions. (Unit cost is zero in Shaked and Sutton (1982) and a quadratic function of quality in Gal-Or (1985).) Mine does not. Instead, I seek more general sufficient and necessary conditions for offering a higher-quality product to be more (less) profitable. Second, quality choices are endogenous in both Shaked and Sutton (1982) and Gal-Or (1985), but are exogenously given in this paper. Even though equilibrium quality location is not a central concern of this model, the results obtained here readily apply once the firms have chosen their quality levels.

My modeling framework is also similar to those of Mussa and Rosen (1978), Gabszewicz and Thisse (1979, 1980), Shaked and Sutton (1983), and Itoh (1983). However, these early classics on vertical differentiation address issues other than ranking the profitability of firms selling products of different qualities. Furthermore, most of these articles also adopt specific forms of cost functions. Assuming a strictly convex unit cost function of quality, Mussa and Rosen (1978) study how a monopolist should price its product line to discriminate against consumers with heterogeneous willingness to pay. The unit costs of producing at different quality levels are assumed to be zero in Gabszewicz and Thisse (1979, 1980). Gabszewicz and Thisse (1980) and Shaked and Sutton (1983) point out an interesting "finiteness" property of industries characterized with vertical differentiation (in contrast to horizontal differentiation): under certain conditions on the cost function the market can only sustain a limited number of firms at a Nash price equilibrium, even in the absence of any fixed costs of entry. In a monopolistic setting 
reminiscent of Mussa and Rosen (1978), Itoh (1983) analyzes how prices of existing products, and consequently consumer welfare, may be affected by introduction of a new product (or finer product differentiation.) Champsaur and Rochet (1989) extend the Mussa-Rosen model to a duopoly where the firms offer non-overlapping, continuous spectrums of qualities.

Prices instead of quantities are the firms' decision variables in the papers just mentioned, except for Gal-Or (1985). Other Cournot-type models of vertical differentiation include Gal-Or (1983), Moorthy (1985), and more recently, De Fraja (1996) and Johnson and Myatt (2003). The article by Johnson and Myatt (2003) examine when a multiproduct monopolist may expand or contract its product line in response to entry. Since the present paper deals with competition between single-product, price-setting firms, this stream of papers are not discussed in full detail here.

A model is presented in Section 2. Section 3 contains the core of this paper. Assuming logconcavity of the consumer distribution, Section 3 first develops the notion of relative cost efficiency and the associated sufficient conditions for a higher-quality product to earn a higher (lower) profit margin. It then proceeds to show that the absolute cost efficiency of a firm is also a useful measure for its profitability. Section 4 looks at the special case of a uniform consumer distribution and derives more detailed results. In particular, the uniform distribution assumption makes it possible to derive sufficient conditions for the equilibrium profits to be monotone in quality. Section 6 concludes.

\section{Model}

There are $K$ firms in this market. Production has constant returns to scale for all firms, and the quality and cost levels of each firm are exogenously given. Specifically, firm $k$ produces a good of quality $v_{k}$ at a constant unit cost $c_{k}>0$, and there are no fixed costs of production. The firms compete through 
setting prices simultaneously. I assume that the quality levels of the firms are all distinct, to avoid trivialities due to the basic Bertrand argument. Without loss of generality I index the firms so that $0<v_{1}<v_{2}<\ldots<v_{K}$. Each firm is thus represented by a point $\left(v_{k}, c_{k}\right)$ in the quality-cost space. Connecting each pair of adjacent points $\left(v_{k-1}, c_{k-1}\right)$ and $\left(v_{k}, c_{k}\right)$ with a straight line would lead to the "cost trajectory" of this oligopoly. Define

$$
d_{k}=\frac{c_{k}-c_{k-1}}{v_{k}-v_{k-1}}, \text { for } k=1, \ldots, K,
$$

where $v_{0}=c_{0}=0$. Here $v_{0}$ is a "virtual" product that costs nothing to produce. Each $d_{k}$ represents the "slope" of the cost trajectory between $v_{k-1}$ and $v_{k}$. Since no restrictions are imposed on the firms' costs (other than $\left.c_{k}>0\right), d_{k}$ need not be monotonic in $k$. Therefore, there may be "zigzags" in the cost trajectory of the oligopoly.

Throughout this paper, a cost trajectory is said to be concave (convex) if $d_{1} \geq d_{2} \geq \ldots \geq d_{K}\left(d_{1} \leq d_{2} \leq \ldots \leq d_{K}\right)$, is $S$-shaped if it is first convex and then concave $\left(d_{1} \leq \ldots \leq d_{k} \geq \ldots \geq d_{K}\right.$ for some $\left.1<k<K\right)$, and is inversed-S-shaped if it is first concave and then convex as quality rises $\left(d_{1} \geq \ldots \geq d_{k} \leq \ldots \leq d_{K}\right.$ for some $\left.1<k<K\right)$. A cost trajectory is said to be locally concave (convex) over a contiguous range of qualities $v_{i}, \ldots, v_{j}$ if $d_{i} \geq \ldots \geq d_{j+1}\left(d_{i} \leq \ldots \leq d_{j+1}\right)$.

Each consumer has a unit demand, i.e., she either purchases precisely one unit from the products offered or does not purchase. Assume that a consumer's utility is multiplicatively separable in product quality and her own type characteristic. Without further loss of generality I adopt the familiar Mussa-Rosen utility function. A multiplicatively separable utility function can be transformed into the Mussa-Rosen form through rescaling (cf. Itoh (1983) and Johnson and Myatt (2003)). Each consumer has a constant marginal willingness to pay for quality, and a consumer of type $\theta$ obtains net utility $\theta v-p$ from a product of quality $v$ priced at $p$. I first derive the core results of this paper assuming that $\theta$ has a logconcave density on $[0,1]$, 
and then examine the case when $\theta$ is uniformly distributed for purposes of illustration and deriving further results.

Denote the price of firm $k$ as $p_{k}$. Connecting each pair of adjacent points $\left(v_{k-1}, p_{k-1}\right)$ and $\left(v_{k}, p_{k}\right)$ in the quality-price space with a straight line, one would obtain the "price trajectory" of this oligopoly. Let

$$
\theta_{k}=\frac{p_{k}-p_{k-1}}{v_{k}-v_{k-1}}, \text { for } k=1, \ldots, K,
$$

where $v_{0}=p_{0}=0$, i.e., the zero-quality product is a free product with zero utility for all consumers. Selecting $v_{0}$ thus amounts to not purchasing at all.

Here $\theta_{k}$ is the "slope" of the price trajectory between firms $k-1$ and $k$. A consumer of type $\theta_{k}$ obtains the same level of net utility from buying $v_{k-1}$ and $v_{k}$, and is therefore indifferent between these two quality levels. A familiar implication of the Mussa-Rosen utility function is that consumers between $\theta_{k}$ and $\theta_{k+1}$ (with $\theta_{K+1}=1$ ) will prefer product $k$, while consumers below $\theta_{1}$ will make no purchase. Therefore, if the firms set prices so that $\theta_{k}$ is non-decreasing in $k$, the demand and profit of firm $k$ are

$$
D_{k}=F\left(\theta_{k+1}\right)-F\left(\theta_{k}\right)
$$

and

$$
\pi_{k}=\left(p_{k}-c_{k}\right)\left(F\left(\theta_{k+1}\right)-F\left(\theta_{k}\right)\right)
$$

respectively. The firms set prices simultaneously to maximize their own profits.

\section{The Consumer Distribution has a Logcon- cave Density}

Suppose that the density of consumer types is $f(\theta)$ on $[0,1]$, with $\int_{0}^{1} f(\theta) d \theta=$ 1. Let $F(\theta)=\int_{0}^{\theta} f(x) d x$. 
Assumption 1. $f(\theta)$ is logconcave and differentiable on $(0,1)$.

Many frequently used distributions have logconcave densities, e.g., the exponential, normal, uniform, and Weibull distributions, among others. By a result due to Caplin and Nalebuff (1991), under Assumption 1 a Nash equilibrium exists in the pricing game described in the Model section. Caplin and Nalebuff (1991) consider a more general setting of imperfect competition that encompasses vertical differentiation, and show that a log-concave density of consumer types ensures the existence of price equilibrium.

Apparently, the firms in the present model have no incentive to price below cost. This paper focuses on the situation where all $K$ firms in the oligopoly are active at equilibrium, in the sense that each firm attracts strictly positive demand at a price at or above its unit cost.

The first-order condition (FOC) of firm $k(k<K)$ is

$$
F\left(\theta_{k+1}\right)-F\left(\theta_{k}\right)=\left(p_{k}-c_{k}\right)\left[\frac{f\left(\theta_{k+1}\right)}{v_{k+1}-v_{k}}+\frac{f\left(\theta_{k}\right)}{v_{k}-v_{k-1}}\right],
$$

where $v_{0}=0$, and the FOC of firm $K$ is

$$
1-F\left(\theta_{K}\right)=\left(p_{K}-c_{K}\right) \frac{f\left(\theta_{K}\right)}{v_{K}-v_{K-1}} .
$$

Firm $k$ 's FOC equates the marginal benefit to the marginal cost of a unit increase in its price $p_{k}$. The LHS of the $k$ th equation above is the demand of firm $k$, and represents the profit increase from the consumers who continue to purchase from firm $k$ after the unit increase in its price. On the other hand, a unit increase in $p_{k}(k<K)$ also lowers $\theta_{k+1}$ by $1 /\left(v_{k+1}-v_{k}\right)$ and raises $\theta_{k}$ by $1 /\left(v_{k}-v_{k-1}\right)$, thus causing firm $k$ 's initial consumers in the number of $f\left(\theta_{k+1}\right) /\left(v_{k+1}-v_{k}\right)$ and $f\left(\theta_{k}\right) /\left(v_{k}-v_{k-1}\right)$ to switch to firms $k+1$ and $k-1$, respectively. Likewise, a unit increase in $p_{K}$ will cause $f\left(\theta_{K}\right) /\left(v_{K}-v_{K-1}\right)$ 
of firm $K$ 's consumers to switch to firm $K-1$. The RHS thus stands for firm $k$ 's profit loss due to lost consumers after the unit price increase. At equilibrium firm $k$ obtains positive demand as long as $p_{k}>c_{k}$. Since this paper assumes that each firm obtains positive sales, at equilibrium $\theta_{k}$ must be strictly increasing in $k$. That is, the equilibrium price trajectory is piecewise linear, increasing and convex.

Define

$$
G(x, y \mid \alpha, \beta) \equiv \frac{F(x)-F(y)}{\alpha f(x)+\beta f(y)}, \text { for } 0<y<x<1 \text { and } \alpha, \beta>0,
$$

and

$$
H(y \mid \beta) \equiv \frac{1-F(y)}{\beta f(y)}, \text { for } 0<y<1 \text { and } \beta>0 .
$$

Lemma 1 Under Assumption 1, (1) $G(x, y \mid \alpha, \beta)$ is increasing in $x$ and decreasing in $y$, for $0<y<x<1$; and (2) $H(y \mid \beta)$ is non-increasing in $y$, for $0<y<1$.

Proof: Under Assumption 1, $f(x)>0$ and $f^{\prime}(x) / f(x)$ is nonincreasing on $(0,1)$. A strictly positive density on the interior of its support implies that $F(x)$ is strictly increasing on $[0,1]$.

(1) It is straightforward to verify that

$$
\begin{aligned}
\frac{\partial}{\partial x} G(x, y \mid \alpha, \beta) & >0 \\
& \Longleftrightarrow f(x)[\alpha f(x)+\beta f(y)]-\alpha f^{\prime}(x)[F(x)-F(y)]>0 \\
& \Longleftrightarrow \frac{f^{\prime}(x)}{f(x)}<\frac{\alpha f(x)+\beta f(y)}{\alpha[F(x)-F(y)]}
\end{aligned}
$$

and that

$$
\frac{\alpha f(x)+\beta f(y)}{\alpha[F(x)-F(y)]}>\frac{f(x)}{F(x)} .
$$

Therefore, to show $G(x, y \mid \alpha, \beta)$ is increasing in $x$, we only need to show 
$f^{\prime}(x) / f(x) \leq f(x) / F(x)$, which holds because

$$
\frac{f(x)}{F(x)}=\frac{\int_{0}^{x} f^{\prime}(z) d z}{\int_{0}^{x} f(z) d z}=\frac{\int_{0}^{x}\left[f^{\prime}(z) / f(z)\right] f(z) d z}{\int_{0}^{x} f(z) d z} \geq \frac{f^{\prime}(x)}{f(x)} .
$$

We can also verify that

$$
\begin{aligned}
\frac{\partial}{\partial y} G(x, y \mid \alpha, \beta) & <0 \\
& \Longleftrightarrow-f(y)[\alpha f(x)+\beta f(y)]-\beta f^{\prime}(y)[F(x)-F(y)]<0 \\
& \Longleftrightarrow \frac{f^{\prime}(y)}{f(y)}>-\frac{\alpha f(x)+\beta f(y)}{\beta[F(x)-F(y)]}
\end{aligned}
$$

and that

$$
-\frac{\alpha f(x)+\beta f(y)}{\beta[F(x)-F(y)]}<\frac{-f(y)}{F(x)-F(y)}<\frac{-f(y)}{1-F(y)} .
$$

Therefore, $G(x, y \mid \alpha, \beta)$ is decreasing in $y$ if $f^{\prime}(y) / f(y) \geq-f(y) /(1-$ $F(y)$ ), which follows from

$$
\frac{-f(y)}{1-F(y)} \leq \frac{f(1)-f(y)}{1-F(y)}=\frac{\int_{y}^{1} f^{\prime}(z) d z}{\int_{y}^{1} f(z) d z}=\frac{\int_{y}^{1}\left[f^{\prime}(z) / f(z)\right] f(z) d z}{\int_{y}^{1} f(z) d z} \leq \frac{f^{\prime}(y)}{f(y)} .
$$

(2) We can verify that $H^{\prime}(y \mid \beta) \leq 0$ if and only if $-f^{2}(y)-f^{\prime}(y)[1-F(y)] \leq$ 0 , or equivalently $f^{\prime}(y) / f(y) \geq-f(y) /(1-F(y))$, which holds as just shown above. Q.E.D.

Let $m_{k}=p_{k}-c_{k}$ and

$$
r_{k}=\frac{1}{v_{k}-v_{k-1}} .
$$

Here $1 / r_{k}$ measures the degree of product differentiation between firm $k$ and its immediate downstream rival, firm $k-1$.

Rewriting (5) and (6) in terms of profit margins $m_{k}$ and $r_{k}$ will greatly 
ease the subsequent analysis. For firms $k<K$,

$$
\begin{aligned}
& F\left(\left(m_{k+1}-m_{k}\right) r_{k+1}+d_{k+1}\right)-F\left(\left(m_{k}-m_{k-1}\right) r_{k}+d_{k}\right) \\
= & m_{k}\left[f\left(\left(m_{k+1}-m_{k}\right) r_{k+1}+d_{k+1}\right) r_{k+1}+f\left(\left(m_{k}-m_{k-1}\right) r_{k}+d_{k}\right) r_{k}\right]
\end{aligned}
$$

where $m_{0}=v_{0}=0$, and for firm $K$,

$$
1-F\left(\left(m_{K}-m_{K-1}\right) r_{K}+d_{K}\right)=m_{K} f\left(\left(m_{K}-m_{K-1}\right) r_{K}+d_{K}\right) r_{K} .
$$

Proposition 2 identifies a sufficient condition for the firms' profit margins to be monotone increasing in the qualities of their products.

Proposition 2 Under Assumption 1, suppose

$$
m_{1}^{\prime} \leq G\left(d_{3}, d_{2} \mid r_{3}, r_{2}\right) \leq \ldots \leq G\left(d_{K}, d_{K-1} \mid r_{K}, r_{K-1}\right) \leq H\left(d_{K} \mid r_{K}\right)
$$

where $m_{1}^{\prime}$ is the unique solution to $m_{1}^{\prime}=G\left(d_{2}, d_{1}+m_{1}^{\prime} r_{1} \mid r_{2}, r_{1}\right)$. Then $m_{1} \leq$ $\ldots \leq m_{K}$.

Proof: First, we show the uniqueness of $m_{1}^{\prime}$. Let $W\left(m_{1}\right)=m_{1}$. Clearly, $W\left(m_{1}\right)$ is strictly increasing. From Lemma $1, G\left(d_{2}, d_{1}+m_{1} r_{1} \mid r_{2}, r_{1}\right)$ is strictly decreasing in $m_{1}$. The remaining argument to establish the uniqueness of $m_{1}^{\prime}$ splits into three cases. Case (1): when $d_{2}>d_{1}$. At $m_{1}=0, W\left(m_{1}\right)=0$ and $G\left(d_{2}, d_{1}+m_{1} r_{1} \mid r_{2}, r_{1}\right)>0$. At $m_{1}=\left(d_{2}-d_{1}\right) v_{1}, W\left(m_{1}\right)>0$ and $G\left(d_{2}, d_{1}+m_{1} r_{1} \mid r_{2}, r_{1}\right)=0$. Therefore $m_{1}^{\prime}>0$ must be unique. Case (2): when $d_{2}<d_{1}$. At $m_{1}=0, W\left(m_{1}\right)=0$ and $G\left(d_{2}, d_{1}+m_{1} r_{1} \mid r_{2}, r_{1}\right)<0$. At $m_{1}=\left(d_{2}-d_{1}\right) v_{1}, W\left(m_{1}\right)<0$ and $G\left(d_{2}, d_{1}+m_{1} r_{1} \mid r_{2}, r_{1}\right)=0$. Therefore $m_{1}^{\prime}<0$ must be unique. Case (3): when $d_{2}=d_{1}$. In this case, at $m_{1}>0$, $W\left(m_{1}\right)>0$ but $G\left(d_{2}, d_{1}+m_{1} r_{1} \mid r_{2}, r_{1}\right)<0$. At $m_{1}<0, W\left(m_{1}\right)<0$ but $G\left(d_{2}, d_{1}+m_{1} r_{1} \mid r_{2}, r_{1}\right)>0$. Therefore $m_{1}^{\prime}=0$.

The rest of the proof is by induction and has two steps. 
Step 1. We wish to show $m_{1} \leq m_{2}$ under the condition of the Proposition. Suppose $m_{1}>m_{2}$. Then from (7) we have

$$
\begin{aligned}
m_{1} & =G\left(d_{2}+\left(m_{2}-m_{1}\right) r_{2}, d_{1}+m_{1} r_{1} \mid r_{2}, r_{1}\right) \\
& \left.<G\left(d_{2}, d_{1}+m_{1} r_{1} \mid r_{2}, r_{1}\right), \quad \text { (by Lemma } 1\right)
\end{aligned}
$$

which implies

$$
m_{1}<m_{1}^{\prime} .
$$

If $d_{2} \leq d_{1}$, the last inequality implies $m_{1}<0$. A contradiction to $m_{1}>0$ for firm 1 to be active at equilibrium, and thus $m_{1} \leq m_{2}$ must hold.

If $d_{2}>d_{1}$, setting $k=2$ in (7) gives

$$
\begin{aligned}
m_{2} & =G\left(d_{3}+\left(m_{3}-m_{2}\right) r_{3}, d_{2}+\left(m_{2}-m_{1}\right) r_{2} \mid r_{3}, r_{2}\right) \\
& \left.>G\left(d_{3}+\left(m_{3}-m_{2}\right) r_{3}, d_{2} \mid r_{3}, r_{2}\right) . \quad \text { (by Lemma } 1\right)
\end{aligned}
$$

Therefore, we have

$$
\begin{aligned}
& G\left(d_{3}+\left(m_{3}-m_{2}\right) r_{3}, d_{2} \mid r_{3}, r_{2}\right) \\
< & m_{2}<m_{1}<m_{1}^{\prime} \\
= & G\left(d_{2}, d_{1}+m_{1}^{\prime} r_{1} \mid r_{2}, r_{1}\right) \quad \text { (by construction) } \\
\leq & G\left(d_{3}, d_{2} \mid r_{3}, r_{2}\right), \quad \text { (by assumption) }
\end{aligned}
$$

or,

$$
G\left(d_{3}+\left(m_{3}-m_{2}\right) r_{3}, d_{2} \mid r_{3}, r_{2}\right)<G\left(d_{3}, d_{2} \mid r_{3}, r_{2}\right) .
$$

By Lemma 1, the last inequality implies $m_{3}<m_{2}$. Similarly, we can show that when $m_{1}>m_{2}, m_{k}<m_{k-1}$ for all successively higher $k$. That is,

$$
m_{K}<\ldots<m_{1}<m_{1}^{\prime}
$$


However, $m_{K}<m_{K-1}$ and (8) jointly imply

$$
\begin{aligned}
m_{K} & =H\left(d_{K}+\left(m_{K}-m_{K-1}\right) r_{K} \mid r_{K}\right) \\
& \geq H\left(d_{K} \mid r_{K}\right) . \quad(\text { by Lemma } 1)
\end{aligned}
$$

We thus have

$$
H\left(d_{K} \mid r_{K}\right)<m_{1}^{\prime} .
$$

A contradiction to the condition of the Proposition. This proves $m_{1} \leq m_{2}$, completing Step 1.

Step 2. Suppose $m_{k-1} \leq m_{k}(k<K)$ holds, and we wish to show $m_{k} \leq$ $m_{k+1}$ under the condition of the Proposition. This step is very analogous to Step 1, and is relegated to the Appendix. $\quad$ Q.E.D.

To see the rationale behind Proposition 2, it is helpful to examine the economic interpretation of each term in its condition first. The $k$ 'th term $(1<$ $k<K)$ in the condition of Proposition 2 is $\left[F\left(d_{k+1}\right)-F\left(d_{k}\right)\right] /\left[f\left(d_{k+1}\right) r_{k+1}+\right.$ $f\left(d_{k}\right) r_{k}$ ]. Even though this ratio may take negative values (when $d_{k+1}<d_{k}$ ), for now I only focus on the case when it is positive. The numerator of this ratio is firm $k$ 's market size if all firms were to price at their unit costs, and represents the profit increase resulting from a unit price increase by firm $k$. The denominator is the number of firm $k$ 's consumers that would be lost to its competitors due to a unit increase in $p_{k}$. The magnitude of this ratio is thus a measure of firm $k$ 's profitability. Note that this term incorporates both demand- and supply-side factors. In particular, since on the supply side it takes into account the unit costs and quality levels of both firm $k$ and its immediate competitors (namely, firms $k-1$ and $k+1$ ), this term is called firm $k$ 's relative cost efficiency. The corresponding terms for firms 1 and $K$ have similar meanings, with the only difference being that these two firms each have only one direct competitor.

Proposition 2 may thus be interpreted as follows: When the relative cost 
efficiency is increasing in product quality, so are the equilibrium profit margins of the firms. Such an interpretation becomes much more concrete for the special case in which consumer types are uniformly distributed.

Corollary 3 Suppose consumer types are uniformly distributed on [0,1]. If

$$
\frac{d_{2}-d_{1}}{2 r_{1}+r_{2}} \leq \frac{d_{3}-d_{2}}{r_{3}+r_{2}} \leq \ldots \leq \frac{d_{K}-d_{K-1}}{r_{K}+r_{K-1}} \leq \frac{1-d_{K}}{r_{K}}
$$

then $m_{1} \leq m_{2} \leq \ldots \leq m_{K}$

The $k$ th term $(1<k<K)$ in the condition of the Corollary is the change in the slope of the cost trajectory at firm $k\left(d_{k+1}-d_{k}\right)$ multiplied by a parameter reflecting the degree of product differentiation between firms $k-1, k$, and $k+1\left(1 /\left(r_{k+1}+r_{k}\right)\right)$. After some algebra, this term can be rewritten as

$$
\frac{d_{k+1}-d_{k}}{r_{k+1}+r_{k}}=\frac{1}{v_{k+1}-v_{k-1}}\left[\left(v_{k}-v_{k-1}\right) c_{k+1}+\left(v_{k+1}-v_{k}\right) c_{k-1}\right]-c_{k}
$$

The first term in this expression is the quality-weighted average cost of firms $k-1$ and $k+1$. Therefore, $\left(d_{k+1}-d_{k}\right) /\left(r_{k+1}+r_{k}\right)$ represents the qualityweighted cost advantage of firm $k$ relative to the two firms with which it directly competes. Similarly, $\left(d_{2}-d_{1}\right) /\left(2 r_{1}+r_{2}\right)$ reflects firm 1's qualityweighted cost advantage relative to firm 2 , and $\left(1-d_{K}\right) / r_{K}$ reflects firm $K$ 's cost-quality position relative to firm $K-1$.

The condition in Proposition 2 is sufficient, but not necessary for a higher quality product to generate a higher profit margin. For example, a concave cost trajectory may demonstrate decreasing relative cost efficiency (thus violating the condition in Proposition 2) but always ensures increasing profit margins (by Proposition 7 below). It is worthwhile pointing out that the condition in Proposition 2 may be satisfied by concave, convex, or inversedS-shaped cost trajectories. 
Proposition 4 Under Assumption 1, suppose

$$
m_{1}^{\prime} \geq G\left(d_{3}, d_{2} \mid r_{3}, r_{2}\right) \geq \ldots \geq G\left(d_{K}, d_{K-1} \mid r_{K}, r_{K-1}\right) \geq H\left(d_{K} \mid r_{K}\right),
$$

where $m_{1}^{\prime}$ is the unique solution to $m_{1}^{\prime}=G\left(d_{2}, d_{1}+m_{1}^{\prime} r_{1} \mid r_{2}, r_{1}\right)$. Then $m_{1} \geq$ $\ldots \geq m_{K}$.

Its proof closely parallels that of the previous Proposition, and is omitted. According to Proposition 4, the profit margins are monotone decreasing in product quality if each firm offering a successively higher quality product has a lower relative cost efficiency. Note that the condition in Proposition 4 may be satisfied by convex or S-shaped cost trajectories.

Adding the $K$ FOCs in (7) and (8) gives

$$
\begin{aligned}
1-F\left(m_{1} r_{1}+d_{1}\right)= & m_{1} f\left(m_{1} r_{1}+d_{1}\right) r_{1} \\
& +\sum_{2}^{K}\left(m_{k}+m_{k-1}\right) f\left(\left(m_{k}-m_{k-1}\right) r_{k}+d_{k}\right) r_{k} .
\end{aligned}
$$

When the firms obtain equal profit margin, i.e., $m_{1}=\ldots=m_{K}=m^{*}$, (9) becomes

$$
1-F\left(m^{*} r_{1}+d_{1}\right)=m^{*}\left(f\left(m^{*} r_{1}+d_{1}\right) r_{1}+2 \sum_{2}^{K} f\left(d_{k}\right) r_{k}\right) .
$$

By Lemma $1, m^{*}$ is unique. Here $m^{*}$ is the mean profit margin in the industry. Not surprisingly, $m^{*}$ decreases when the products are less differentiated (i.e., the $r_{k}$ 's are larger) or when the number of firms $K$ increases.

Proposition 5 Under Assumption 1, a necessary and sufficient condition for $m_{1}=\ldots=m_{K}=m^{*}$ is

$$
m_{1}^{\prime}=G\left(d_{3}, d_{2} \mid r_{3}, r_{2}\right)=\ldots=G\left(d_{K}, d_{K-1} \mid r_{K}, r_{K-1}\right)=H\left(d_{K} \mid r_{K}\right),
$$

where $m_{1}^{\prime}$ is the unique solution to $m_{1}^{\prime}=G\left(d_{2}, d_{1}+m_{1}^{\prime} r_{1} \mid r_{2}, r_{1}\right)$. 
Proof: (1)Sufficiency. Since the condition of the Proposition satisfies those in both Propositions 2 and 4 , we have $m_{1} \leq \ldots \leq m_{K}$ and $m_{1} \geq \ldots \geq$ $m_{K}$, and hence $m_{1}=\ldots=m_{K}=m^{*}$.

(2)Necessity. Suppose $m_{1}=\ldots=m_{K}=m^{*}$. Then the system of FOCs in (7) and (8) reduces to

$$
\begin{gathered}
m^{*}=G\left(d_{2}, d_{1}+m^{*} r_{1} \mid r_{2}, r_{1}\right), \\
m^{*}=G\left(d_{k+1}, d_{k} \mid r_{k+1}, r_{k}\right), \text { for } 1<k<K,
\end{gathered}
$$

and

$$
m^{*}=H\left(d_{K} \mid r_{K}\right) .
$$

The desired set of conditions then follows by noting that $m^{*}=m_{1}^{\prime}$. Q.E.D.

When the firms obtain equal profit margin, the cost trajectory of the oligopoly must be increasing and convex. It is readily verified that, when $m_{1}=\ldots=m_{K}=m^{*}$, the equilibrium demands of the firms are $D_{1}=$ $F\left(d_{2}\right)-F\left(m^{*} r_{1}+d_{1}\right), D_{k}=F\left(d_{k+1}\right)-F\left(d_{k}\right)($ for $1<k<K)$, and $D_{K}=$ $1-F\left(d_{K}\right)$.

Next, I proceed to show that the ratio between a firm's quality level and its unit cost $\left(v_{k} / c_{k}\right)$ is also a useful measure for its profitability. The ratio $v_{k} / c_{k}$ is called firm $k$ 's absolute cost efficiency in quality provision. Proposition 6 below says that a firm capable of producing a higher quality product at a higher absolute cost efficiency earns a higher profit.

Proposition 6 If $c_{i} / v_{i} \geq c_{j} / v_{j}$ for $1 \leq i<j \leq K$, then $m_{i}<m_{j}$ and $\pi_{i}<\pi_{j}$.

Proof: Suppose $\left(p_{1}, \ldots, p_{K}\right)$ is the unique price equilibrium. This means, when the remaining $K-1$ firms price at $\left(p_{1}, \ldots, p_{j-1}, p_{j+1}, \ldots, p_{K}\right)$, firm $j$ 's best choice is $p_{j}$. Recall that when all $K$ firms are active, the equilibrium price 
trajectory is a piecewise linear, increasing, and convex function of quality, i.e., $\theta_{k}<\theta_{k+1}$, for $k<K$. This implies $\left(p_{j}-p_{i}\right) /\left(v_{j}-v_{i}\right)>p_{i} / v_{i}$.

Note that the condition of the Proposition is equivalent to $c_{i} / v_{i} \geq\left(c_{j}-\right.$ $\left.c_{i}\right) /\left(v_{j}-v_{i}\right)$. Since $p_{i} / v_{i}>c_{i} / v_{i}$, we have $\left(p_{j}-p_{i}\right) /\left(v_{j}-v_{i}\right)>\left(c_{j}-c_{i}\right) /\left(v_{j}-v_{i}\right)$, or equivalently $m_{i}<m_{j}$. Note that firm $j$ has the option to lower its price to $p_{j}^{\prime}=c_{j}+m_{i}$. Because

$$
\frac{p_{j}^{\prime}-p_{i}}{v_{j}-v_{i}}=\frac{c_{j}-c_{i}}{v_{j}-v_{i}} \leq \frac{c_{i}}{v_{i}}<\frac{p_{i}}{v_{i}}
$$

at price $p_{j}^{\prime}$ firm $j$ would take over the entire demand of firms $i, \ldots, j-1$ (plus possibly the demand of some other firms). Therefore, at price $p_{j}^{\prime}$ firm $j$ 's profit would clearly exceed firm $i$ 's equilibrium profit $\pi_{i}$. However $p_{j}^{\prime}$ is a price firm $j$ does not choose. Therefore we must have $\pi_{i}<\pi_{j}$. Q.E.D.

The two firms compared in Proposition 6 need not be directly competing. The key argument in the proof above is the following. Because the equilibrium price trajectory is increasing and convex, under the condition of the Proposition, firm $j$ (the firm offering a higher quality) always obtains a higher profit margin than firm $i$, i.e., $m_{j}>m_{i}$. If firm $j$ chose to price at $c_{j}+m_{i}$ it could have attracted away the entire demand of firm $i$ and thus made a profit exceeding firm $i$ 's equilibrium profit. However firm $j$ does not choose such a price. Therefore, firm $j$ 's equilibrium profit must be higher than that of firm $i$.

Proposition 7 Suppose $c_{1} / v_{1} \geq c_{2} / v_{2} \geq \ldots \geq c_{K} / v_{K}$. Then $m_{1}<\ldots<m_{K}$ and $\pi_{1}<\ldots<\pi_{K}$.

Proposition 7 is an immediate corollary of Proposition 6. Since the proof of Proposition 6 does not depend on the logconcavity assumption (Assumption 1), these two Propositions hold for more general classes of consumer distributions. Since $c_{1} / v_{1} \geq c_{2} / v_{2} \geq \ldots \geq c_{K} / v_{K}$ is equivalent to $v_{1} / c_{1} \leq v_{2} / c_{2}$ $\leq \ldots \leq v_{K} / c_{K}$, Proposition 7 says that when the absolute cost efficiency 
does not decrease as quality rises, firms selling higher quality products always make strictly greater profits. A cost trajectory that demonstrates nondecreasing absolute cost efficiency may embody arbitrary curvatures, except that it can not be strictly convex (where the absolute cost efficiency is decreasing). In particular, a concave cost trajectory $\left(d_{1} \geq d_{2} \ldots \geq d_{K}\right)$ always has non-decreasing absolute cost efficiency.

\section{The Consumer Distribution is Uniform: a Special Case}

This Section examines the uniform consumer distribution as a special case of distributions with logconcave densities.

Assumption 2. Consumer types are uniformly distributed on $[0,1]$.

Henceforward, Assumption 2 replaces Assumption 1. Under Assumption 2 , the first order conditions in (5) and (6) reduce to

$$
\begin{gathered}
\theta_{k+1}-\theta_{k}=\left(p_{k}-c_{k}\right)\left(r_{k+1}+r_{k}\right), \text { for } k \leq K-1, \\
1-\theta_{K}=\left(p_{K}-c_{K}\right) r_{K}, \text { for } k=K,
\end{gathered}
$$

and (7) and (8) now become: For $k<K$,

$$
\begin{gathered}
\left(m_{k+1}-m_{k}\right) r_{k+1}=\left(m_{k}-m_{k-1}\right) r_{k}+m_{k}\left(r_{k+1}+r_{k}\right)-\left(d_{k+1}-d_{k}\right), \\
0=\left(m_{K}-m_{K-1}\right) r_{K}+m_{K} r_{K}-\left(1-d_{K}\right) .
\end{gathered}
$$

As seen in Section 3, Propositions 2, 4 and 5 have their apparent counterparts in the setting of a uniform distribution (cf. Corollary 3), which have 
more intuitive economic interpretations. Here I only deal with a necessary condition of monotone profit margins, which involves a set of bounds of each firm's profit margin.

Proposition 8 Under Assumption 2, suppose $m_{1} \lesseqgtr m_{2} \lesseqgtr \ldots \lesseqgtr m_{K}$ at equilibrium. Then for $1 \leq k \leq K$,

$$
\frac{d_{k+1}-d_{1}}{2 \sum_{1}^{k} r_{i}+r_{k+1}} \lesseqgtr m_{k} \lesseqgtr \frac{1-d_{k}}{r_{k}+2 \sum_{k+1}^{K} r_{i}} .
$$

Proof: The proof is done only for the case in which $m_{1} \leq \ldots \leq m_{K}$, the other case being analogous.

Adding the FOCs of firms $1, \ldots, k$ in (13) and (14) gives

$$
\left(m_{k+1}-m_{k}\right) r_{k+1}=m_{1} r_{1}+\sum_{1}^{k} m_{i}\left(r_{i+1}+r_{i}\right)-\left(d_{k+1}-d_{1}\right) \geq 0,
$$

from which the lower bound of $m_{k}$ follows. Adding the FOCs of firms $k, \ldots, K$ in (13) and (14) and slightly rearranging, we have

$$
\sum_{k}^{K-1} m_{i}\left(r_{i+1}+r_{i}\right)+m_{K} r_{K}=1-d_{k}-\left(m_{k}-m_{k-1}\right) r_{k} \leq 1-d_{k} .
$$

The upper bound of $m_{k}$ then follows from this inequality. Q.E.D.

So far, I have primarily focused on profit margins as a measure of firms' per-unit profitability. Next, I will turn to profits, and identify sufficient conditions under which a firm offering a higher quality product makes a higher (lower) profit.

The first step is to translate the first-order conditions into expressions of firms' profits, instead of profit margins. From (11) and (12), at equilibrium firm k's demand and profit are $D_{k}=m_{k}\left(r_{k+1}+r_{k}\right)$ and $\pi_{k}=m_{k}^{2}\left(r_{k+1}+r_{k}\right)$, 
respectively, where $r_{K+1}=0$. Therefore,

$$
m_{k}=\sqrt{\frac{\pi_{k}}{r_{k+1}+r_{k}}}, \text { where } r_{K+1}=0
$$

With (15), the system of FOCs in (13) and (14) can be rewritten in terms of $\pi_{k}$ :

$$
r_{k+1} \sqrt{\frac{\pi_{k+1}}{r_{k+2}+r_{k+1}}}=2 \sqrt{\left(r_{k+1}+r_{k}\right) \pi_{k}}-r_{k} \sqrt{\frac{\pi_{k-1}}{r_{k}+r_{k-1}}}-\left(d_{k+1}-d_{k}\right),
$$

for firms $k \leq K-1$, where $\pi_{0}=0$ and $r_{0}=0$, and

$$
0=2 \sqrt{r_{K} \pi_{K}}-r_{K} \sqrt{\frac{\pi_{K-1}}{r_{K}+r_{K-1}}}-\left(1-d_{K}\right), \text { for firm } K .
$$

Equations (16)-(17) allow direct manipulation of firms' profits, and are the basis for deriving the next Proposition.

Let

$$
\begin{gathered}
t_{1} \equiv 2 \sqrt{r_{2}+r_{1}}-\frac{r_{2}}{\sqrt{r_{3}+r_{2}}}, \\
t_{k} \equiv 2 \sqrt{r_{k+1}+r_{k}}-\frac{r_{k}}{\sqrt{r_{k}+r_{k-1}}}-\frac{r_{k+1}}{\sqrt{r_{k+2}+r_{k+1}}},
\end{gathered}
$$

for $1<k<K$, where $r_{K+1}=0$, and

$$
t_{K} \equiv 2 \sqrt{r_{K}}-\frac{r_{K}}{\sqrt{r_{K}+r_{K-1}}}
$$

\section{Lemma 9}

$$
\begin{gathered}
\sqrt{r_{k+1}+r_{k}}>\frac{r_{k}}{\sqrt{r_{k}+r_{k-1}}}, \text { for } 2 \leq k \leq K-1, \\
\sqrt{r_{k+1}+r_{k}}>\frac{r_{k+1}}{\sqrt{r_{k+2}+r_{k+1}}}, \text { for } 1 \leq k \leq K-1 .
\end{gathered}
$$


Proof: See Appendix A2.

An immediate implication of Lemma 9 is that $t_{k}>0,1 \leq k<K$. That $t_{K}>0$ is obvious. Just like $r_{k}$, each $t_{k}$ measures the degree of differentiation between firm $k$ 's product $v_{k}$ and those of its neighboring firms, except that $t_{k}$ takes into account the qualities of up to two (instead of one) firms below and above $v_{k}$.

As the next Proposition shows, when $\left(d_{k+1}-d_{k}\right) / t_{k}\left(\right.$ with $\left.d_{K+1}=1\right)$ is monotone in $k$, so is equilibrium profit $\pi_{k}$.

Proposition 10 Under Assumption 2, if

$$
\frac{d_{2}-d_{1}}{t_{1}} \lesseqgtr \ldots \lesseqgtr \frac{d_{K}-d_{K-1}}{t_{K-1}} \lesseqgtr \frac{1-d_{K}}{t_{K}},
$$

then $\pi_{1} \lesseqgtr \ldots \lesseqgtr \pi_{K}$.

Proof: See Appendix A3.

Even though its composition is too complex to allow a direct interpretation, each term in the condition of the Proposition $\left(\left(d_{k+1}-d_{k}\right) / t_{k}\right)$ may still be viewed as a measure of firm $k$ 's relative cost efficiency, because it takes into consideration the costs and qualities of both firm $k$ itself and its adjacent competing firms. When a firm can produce a higher quality product more efficiently according to this measure, it also obtains greater profit at equilibrium.

Under Assumption 2, (9) may also be rewritten (with (15)) as

$$
1-d_{1}=r_{1} \sqrt{\frac{\pi_{1}}{r_{2}+r_{1}}}+\sum_{1}^{K-1} \sqrt{\pi_{k}\left(r_{k+1}+r_{k}\right)}+\sqrt{\pi_{K} r_{K}} .
$$

If all $K$ firms earn the same level of profit, $\pi^{*}$ say, then (18) leads to

$$
\sqrt{\pi^{*}}=\frac{1-d_{1}}{M}
$$


where

$$
M=\frac{r_{1}}{\sqrt{r_{2}+r_{1}}}+\sum_{1}^{K-1} \sqrt{r_{k+1}+r_{k}}+\sqrt{r_{K}} .
$$

A necessary and sufficient condition for the firms to attain the same level of profit is that their relative cost efficiencies $\left(\left(d_{k+1}-d_{k}\right) / t_{k}\right)$ are equal.

Proposition 11 Under Assumption 2, the $K$ firms obtain equal profit at equilibrium if and only if

$$
d_{k+1}-d_{k}=\sqrt{\pi^{*}} \cdot t_{k}, \text { for } 1 \leq k \leq K
$$

Proof: (Necessity) Suppose that all $K$ firms have the same profit. Then as shown above, this common profit level $\pi^{*}$ is as given in (19). Setting $\pi_{k}=\pi^{*}$ in (16)-(17) and slightly rearranging yield the desired conditions.

(Sufficiency) Suppose the condition of the Proposition holds. The previous Proposition immediately lead to $\pi_{1}=\ldots=\pi_{K}$. The common profit level $\pi^{*}$ then follows from (19). Q.E.D.

Proposition 12 Suppose $d_{i} \geq d_{i+1} \geq \ldots \geq d_{j}$, for $1 \leq i<j \leq K$. Then either $\pi_{k}<\pi_{i-1}$ or $\pi_{k}<\pi_{j}$, for $i \leq k \leq j-1$.

Proof: The proof critically relies on the following claim.

Claim 1: Suppose $d_{k} \geq d_{k+1}$, for $1<k<K$. Then either $\pi_{k}<\pi_{k-1}$ or $\pi_{k}<\pi_{k+1}$.

Proof of Claim 1: Suppose $\pi_{k} \geq \pi_{k-1}$ and $\pi_{k} \geq \pi_{k+1}$. We then have

$$
\begin{aligned}
r_{k+1} \sqrt{\frac{\pi_{k}}{r_{k+2}+r_{k+1}}} & \geq r_{k+1} \sqrt{\frac{\pi_{k+1}}{r_{k+2}+r_{k+1}}} \\
& =2 \sqrt{\left(r_{k+1}+r_{k}\right) \pi_{k}}-r_{k} \sqrt{\frac{\pi_{k-1}}{r_{k}+r_{k-1}}}-\left(d_{k+1}-d_{k}\right) \\
& \geq 2 \sqrt{\left(r_{k+1}+r_{k}\right) \pi_{k}}-r_{k} \sqrt{\frac{\pi_{k}}{r_{k}+r_{k-1}}}
\end{aligned}
$$


where the equality is due to (16). This implies

$$
2 \sqrt{r_{k+1}+r_{k}} \leq \frac{r_{k}}{\sqrt{r_{k}+r_{k-1}}}+\frac{r_{k+1}}{\sqrt{r_{k+2}+r_{k+1}}}
$$

which contradicts Lemma 9. Therefore either $\pi_{k}<\pi_{k-1}$ or $\pi_{k}<\pi_{k+1}$ must hold. This completes the proof of Claim 1.

When $j=i+1$, the Proposition reduces to Claim 1 .

When $j>i+1$, suppose there exists some $i \leq k \leq j-1$ so that $\pi_{k} \geq \pi_{i-1}$ and $\pi_{k} \geq \pi_{j}$. By Claim 1, either $\pi_{k}<\pi_{k-1}$ or $\pi_{k}<\pi_{k+1}$ holds. If $\pi_{k}<\pi_{k-1}$, then $\pi_{k-1}<\pi_{k-2}$ must hold due to the Claim. Repeatedly applying Claim 1 leads to $\pi_{k}<\pi_{k-1}<\ldots<\pi_{i-1}$, contradicting $\pi_{k} \geq \pi_{i-1}$. Similarly, if $\pi_{k}<\pi_{k+1}$, one can show that $\pi_{k}<\pi_{k+1}<\ldots<\pi_{j}$. Again, this contradicts $\pi_{k} \geq \pi_{j}$. Therefore either $\pi_{k}<\pi_{i-1}$ or $\pi_{k}<\pi_{j}$ must hold for $i \leq k \leq j-1$. This completes the proof. Q.E.D.

According to Proposition 12, if the cost trajectory is locally concave over a contiguous range of products $v_{i}, \ldots, v_{j-1}$, then each firm within this quality range earns strictly less profit than (at least) one of the two firms at the boundary of this quality range (namely, firms $i-1$ and $j$.) Two special cases of Proposition 12 are as follows. First, if the cost trajectory is kinked upwards at firm $k$ (i.e., $d_{k} \geq d_{k+1}$ ), then firm $k$ 's profit is exceeded by that of firm $k-1$ or $k+1$ (cf. Claim 1 in the above proof.) Second, when $i=1$ and $j=K$ (i.e., when $d_{1} \geq \ldots \geq d_{K}$ ), Proposition 12 predicts that $\pi_{1}<\ldots<\pi_{K}$ since $\pi_{0}=0$. As one may recall, this point was also implied by Proposition 7 .

The next Lemma gives a set of lower and upper bounds on the consumer types who are indifferent between each pair of adjacent qualities at equilibrium. 
Lemma 13 At equilibrium, the indifferent consumers $\theta_{k}(1 \leq k \leq K)$ satisfy

$$
\frac{1}{2}\left(d_{1}+d_{k}\right) \equiv l_{k}<\theta_{k}<u_{k} \equiv \frac{1}{2^{K-k+1}}+\sum_{i=k}^{K} \frac{1}{2^{i-k+1}} d_{i} .
$$

Proof: See Appendix A4.

Lemma 13 then implies the following bounds on the equilibrium profit margins.

Proposition 14 At equilibrium,

$$
\max \left(0, \frac{d_{k+1}-d_{k}}{2\left(r_{k+1}+r_{k}\right)}\right)<m_{k}<\frac{u_{k+1}-l_{k}}{r_{k}},
$$

where $r_{K+1}=0$ and $u_{K+1}=1$.

Proof: The proof uses the fact that at equilibrium $m_{k}\left(r_{k+1}+r_{k}\right)=\theta_{k+1}-$ $\theta_{k}$, where $r_{K+1}=0$ and $\theta_{K+1}=1$ (from (11), (12)). The upper bound of $m_{k}$ then follows directly from Lemma 13. Part I of the proof of Lemma 13 (see Appendix A4) also shows that $\theta_{k+1}-\theta_{k}>(1 / 2)\left(d_{k+1}-d_{k}\right)$. This leads to the lower bound of $m_{k}$. Q.E.D.

From Proposition 14 one can readily derive the corresponding bounds on firms' profits, since $\pi_{k}=m_{k}^{2}\left(r_{k+1}+r_{k}\right)$.

\section{Concluding Remarks}

The analysis in this paper has heavily utilized the structure of the FOCs. Each firm's FOC describes the equilibrium relationship between its own strategic choice (price, or equivalently profit margin) and those of its immediate competitor(s), reflecting the nature of localized competition in vertical differentiation. In Section 3, such a structure of the FOCs together with a logconcave density of consumer types makes it possible to compare the profit margins between each pair of firms offering adjacent quality levels. In Section 
4, the system of FOCs and a uniform consumer distribution enable comparison of the profits of two directly competing firms. A key technique of this analysis is to make a connection between the slopes of the cost trajectory and those of the equilibrium price trajectory.

When firms produce goods of distinct qualities at potentially different unit costs, this paper has identified two useful measures of a firm's profitability, namely its absolute and relative cost efficiencies, and the associated sufficient conditions for selling a higher-quality product to be more (or less) profitable. Perhaps the most interesting results obtained are the following. First, the monotonicity of the relative cost efficiency implies that of profit margins (Propositions 2, 4, 5). Second, when consumer types are uniformly distributed, this paper has also provided sufficient conditions for the equilibrium profits to be monotone in quality as well as bounds on each firm's profitability.

\section{Appendix}

\section{A1. Step 2 in the proof of Proposition 2.}

Suppose $m_{k-1} \leq m_{k}(k<K)$ holds, and we wish to show $m_{k} \leq m_{k+1}$ under the condition of the Proposition.

Suppose $m_{k}>m_{k+1}$ instead. From (7), we have

$$
\begin{aligned}
m_{k} & =G\left(d_{k+1}+\left(m_{k+1}-m_{k}\right) r_{k+1}, d_{k}+\left(m_{k}-m_{k-1}\right) r_{k} \mid r_{k+1}, r_{k}\right) \\
& <G\left(d_{k+1}, d_{k} \mid r_{k+1}, r_{k}\right) . \quad(\text { by Lemma 1) }
\end{aligned}
$$

If $d_{k} \geq d_{k+1},(20)$ is already a contradiction to $m_{k}>0$, and thus $m_{k} \leq$ $m_{k+1}$ must hold. Otherwise, replacing $k$ with $k+1$ in (7) gives

$$
\begin{aligned}
m_{k+1} & =G\left(d_{k+2}+\left(m_{k+2}-m_{k+1}\right) r_{k+2}, d_{k+1}+\left(m_{k+1}-m_{k}\right) r_{k+1} \mid r_{k+2}, r_{k+1}\right) \\
& \left.>G\left(d_{k+2}+\left(m_{k+2}-m_{k+1}\right) r_{k+2}, d_{k+1} \mid r_{k+2}, r_{k+1}\right) . \quad \text { (by Lemma } 1\right)
\end{aligned}
$$


We then have

$$
\begin{aligned}
& G\left(d_{k+2}+\left(m_{k+2}-m_{k+1}\right) r_{k+2}, d_{k+1} \mid r_{k+2}, r_{k+1}\right) \\
< & m_{k+1}<m_{k} \\
< & G\left(d_{k+1}, d_{k} \mid r_{k+1}, r_{k}\right) \\
\leq & G\left(d_{k+2}, d_{k+1} \mid r_{k+2}, r_{k+1}\right)
\end{aligned}
$$

where the last inequality follows from the condition of the Proposition.

Therefore,

$$
G\left(d_{k+2}+\left(m_{k+2}-m_{k+1}\right) r_{k+2}, d_{k+1} \mid r_{k+2}, r_{k+1}\right)<G\left(d_{k+2}, d_{k+1} \mid r_{k+2}, r_{k+1}\right),
$$

which implies $m_{k+1}>m_{k+2}$ by Lemma 1 . Similarly, we can show that when $m_{k}>m_{k+1}$

$$
m_{K}<\ldots<m_{k}<G\left(d_{k+1}, d_{k} \mid r_{k+1}, r_{k}\right)
$$

Again, $m_{K}<m_{K-1}$ and (8) jointly imply

$$
m_{K}=H\left(d_{K}+\left(m_{K}-m_{K-1}\right) r_{K} \mid r_{K}\right) \geq H\left(d_{K} \mid r_{K}\right) .
$$

We thus have

$$
H\left(d_{K} \mid r_{K}\right)<G\left(d_{k+1}, d_{k} \mid r_{k+1}, r_{k}\right) .
$$

A contradiction to the condition of the Proposition. This shows that when $m_{k-1} \leq m_{k}(k<K), m_{k}>m_{k+1}$ can never hold, and therefore, we must have $m_{k} \leq m_{k+1}$ under the condition of the Proposition.

This completes Step 2 of the proof of Proposition 2.

\section{A2. Proof of Lemma 9.}


Proof: When $2 \leq k \leq K-1$, the first statement follows from

$$
\begin{aligned}
& \sqrt{r_{k+1}+r_{k}}-\frac{r_{k}}{\sqrt{r_{k}+r_{k-1}}} \\
= & \frac{1}{\sqrt{v_{k}-v_{k-1}}}\left(\sqrt{\frac{v_{k+1}-v_{k-1}}{v_{k+1}-v_{k}}}-\sqrt{\frac{v_{k-1}-v_{k-2}}{v_{k}-v_{k-2}}}\right)>0,
\end{aligned}
$$

because the first (second) term in the bracket is strictly greater (less) than 1.

The second statement of the lemma can be shown similarly. Q.E.D.

\section{A3. Proof of Proposition 10.}

Proof. We prove the Proposition only for the case in which

$$
\frac{d_{2}-d_{1}}{t_{1}} \leq \ldots \leq \frac{d_{K}-d_{K-1}}{t_{K-1}} \leq \frac{1-d_{K}}{t_{K}} .
$$

The other case is analogous. The proof is by induction.

Step 1 . We wish to show $\pi_{1} \leq \pi_{2}$ under the above condition.

Suppose $\pi_{1}>\pi_{2}$. Then (16) gives

$$
\sqrt{\pi_{1}}<\frac{d_{2}-d_{1}}{t_{1}} .
$$

If $d_{2} \leq d_{1},(21)$ is already a contradiction, and thus $\pi_{1} \leq \pi_{2}$ must hold.

Otherwise, we aim to show $\pi_{K}<\ldots<\pi_{2}<\pi_{1}$ when $\pi_{1}>\pi_{2}$. Suppose $\pi_{3} \geq \pi_{2}$. Then from (16) we have

$$
\begin{aligned}
r_{3} \sqrt{\frac{\pi_{2}}{r_{4}+r_{3}}} & \leq r_{3} \sqrt{\frac{\pi_{3}}{r_{4}+r_{3}}} \\
& =2 \sqrt{\left(r_{3}+r_{2}\right) \pi_{2}}-r_{2} \sqrt{\frac{\pi_{1}}{r_{2}+r_{1}}}-\left(d_{3}-d_{2}\right) \\
& <2 \sqrt{\left(r_{3}+r_{2}\right) \pi_{2}}-r_{2} \sqrt{\frac{\pi_{2}}{r_{2}+r_{1}}}-\left(d_{3}-d_{2}\right)
\end{aligned}
$$


which leads to

$$
\sqrt{\pi_{2}}>\frac{d_{3}-d_{2}}{t_{2}}
$$

This and (21) jointly imply

$$
\frac{d_{3}-d_{2}}{t_{2}}<\frac{d_{2}-d_{1}}{t_{1}}
$$

contradicting the condition of the Proposition. Therefore we must have $\pi_{3}<$ $\pi_{2}$.

Similarly, when $\pi_{1}>\pi_{2}$, we can show $\pi_{k}<\pi_{k-1}$ for successively higher $k$. That is, under the supposition that $\pi_{1}>\pi_{2}$,

$$
\sqrt{\pi_{K}}<\ldots<\sqrt{\pi_{1}}<\frac{d_{2}-d_{1}}{t_{1}}
$$

Note that $\pi_{K}<\pi_{K-1}$ and (17) jointly imply

$$
1-d_{K}<\left(2 \sqrt{r_{K}}-r_{K} \sqrt{\frac{1}{r_{K}+r_{K-1}}}\right) \sqrt{\pi_{K}}
$$

or equivalently

$$
\sqrt{\pi_{K}}>\frac{1-d_{K}}{t_{K}}
$$

We thus have

$$
\frac{1-d_{K}}{t_{K}}<\frac{d_{2}-d_{1}}{t_{1}}
$$

A contradiction to the condition of the Proposition. Therefore $\pi_{1}>\pi_{2}$ can not hold. This establishes $\pi_{1} \leq \pi_{2}$, completing step 1 .

Step 2. Suppose $\pi_{k-1} \leq \pi_{k}(k<K)$ holds, and we wish to show $\pi_{k} \leq \pi_{k+1}$ under the condition of the Proposition. 
Suppose $\pi_{k}>\pi_{k+1}$. Then from (16) we have

$$
\begin{aligned}
r_{k+1} \sqrt{\frac{\pi_{k}}{r_{k+2}+r_{k+1}}} & >r_{k+1} \sqrt{\frac{\pi_{k+1}}{r_{k+2}+r_{k+1}}} \\
& =2 \sqrt{\left(r_{k+1}+r_{k}\right) \pi_{k}}-r_{k} \sqrt{\frac{\pi_{k-1}}{r_{k}+r_{k-1}}}-\left(d_{k+1}-d_{k}\right) \\
& \geq 2 \sqrt{\left(r_{k+1}+r_{k}\right) \pi_{k}}-r_{k} \sqrt{\frac{\pi_{k}}{r_{k}+r_{k-1}}}-\left(d_{k+1}-d_{k}\right),
\end{aligned}
$$

which gives

$$
\sqrt{\pi_{k}}<\frac{d_{k+1}-d_{k}}{t_{k}} .
$$

If $d_{2} \leq d_{1}$, (22) is already a contradiction, and thus $\pi_{k} \leq \pi_{k+1}$ must hold.

Otherwise, we aim to show that $\pi_{K}<\ldots<\pi_{k}$ when $\pi_{k}>\pi_{k+1}$. If $\pi_{k+2} \geq \pi_{k+1}$, then it follows from the FOC of firm $k+1$ (replacing $k$ with $k+1$ in (16)) that

$$
\begin{aligned}
r_{k+2} \sqrt{\frac{\pi_{k+1}}{r_{k+3}+r_{k+2}}} & \leq r_{k+2} \sqrt{\frac{\pi_{k+2}}{r_{k+3}+r_{k+2}}} \\
& =2 \sqrt{\left(r_{k+2}+r_{k+1}\right) \pi_{k+1}}-r_{k+1} \sqrt{\frac{\pi_{k}}{r_{k+1}+r_{k}}}-\left(d_{k+2}-d_{k+1}\right) \\
& <2 \sqrt{\left(r_{k+2}+r_{k+1}\right) \pi_{k+1}}-r_{k+1} \sqrt{\frac{\pi_{k+1}}{r_{k+1}+r_{k}}}-\left(d_{k+2}-d_{k+1}\right)
\end{aligned}
$$

or

$$
\sqrt{\pi_{k+1}}>\frac{d_{k+2}-d_{k+1}}{t_{k+1}} .
$$

This and (22) above jointly imply

$$
\frac{d_{k+2}-d_{k+1}}{t_{k+1}}<\frac{d_{k+1}-d_{k}}{t_{k}} \text {. }
$$

A contradiction to the condition of the Proposition. Therefore when $\pi_{k}>$ $\pi_{k+1}$ we must have $\pi_{k+2}<\pi_{k+1}$. 
Similarly we can show that when $\pi_{k}>\pi_{k+1}$,

$$
\sqrt{\pi_{K}}<\ldots<\sqrt{\pi_{k}}<\frac{d_{k+1}-d_{k}}{t_{k}}
$$

However, $\pi_{K}<\pi_{K-1}$ and (17) jointly imply

$$
\sqrt{\pi_{K}}>\frac{1-d_{K}}{t_{K}}
$$

Therefore,

$$
\frac{1-d_{K}}{t_{K}}<\frac{d_{k+1}-d_{k}}{t_{k}}
$$

A contradiction to the condition of the Proposition. Therefore $\pi_{k}>\pi_{k+1}$ can not hold.

We have shown that $\pi_{k-1} \leq \pi_{k}(k<K)$ implies $\pi_{k} \leq \pi_{k+1}$ in Step 2 . This completes the proof of the Proposition. Q.E.D.

\section{A4. Proof of Lemma 13.}

Proof: The proof is divided into two parts. In Part I (II), we establish the lower (upper) bounds on $m_{k}$.

Part I: Lower bounds. For $1 \leq k \leq K-1$, at equilibrium we have

$$
\theta_{k+1}-\theta_{k}=\left(p_{k}-c_{k}\right)\left(\frac{1}{v_{k+1}-v_{k}}+\frac{1}{v_{k}-v_{k-1}}\right)
$$

or equivalently

$$
\frac{\left(p_{k+1}-c_{k}\right)-\left(p_{k}-c_{k}\right)}{v_{k+1}-v_{k}}-\frac{\left(p_{k}-c_{k}\right)+\left(c_{k}-p_{k-1}\right)}{v_{k}-v_{k-1}}=\left(p_{k}-c_{k}\right)\left(\frac{1}{v_{k+1}-v_{k}}+\frac{1}{v_{k}-v_{k-1}}\right) .
$$

Rearranging terms, we have

$$
\left(p_{k}-c_{k}\right)\left(\frac{1}{v_{k+1}-v_{k}}+\frac{1}{v_{k}-v_{k-1}}\right)=\frac{1}{2}\left(\frac{p_{k+1}-c_{k}}{v_{k+1}-v_{k}}-\frac{c_{k}-p_{k-1}}{v_{k}-v_{k-1}}\right)
$$


Therefore,

$$
\begin{aligned}
\theta_{k+1}-\theta_{k} & =\frac{1}{2}\left(\frac{p_{k+1}-c_{k}}{v_{k+1}-v_{k}}-\frac{c_{k}-p_{k-1}}{v_{k}-v_{k-1}}\right) \\
& \left.>\frac{1}{2}\left(\frac{c_{k+1}-c_{k}}{v_{k+1}-v_{k}}-\frac{c_{k}-c_{k-1}}{v_{k}-v_{k-1}}\right) \quad \text { (since } p_{i}>c_{i}\right) \\
& =\frac{1}{2}\left(d_{k+1}-d_{k}\right),
\end{aligned}
$$

or

$$
\theta_{k+1}>\theta_{k}+\frac{1}{2}\left(d_{k+1}-d_{k}\right)
$$

Since $\theta_{1}>l_{1} \equiv d_{1}$ holds trivially (due to $p_{1}>c_{1}$ ), the lower bounds of the remaining $\theta_{k}$ 's are then derived by induction for successively higher $k$ :

$$
\theta_{k}>l_{k} \equiv \frac{1}{2}\left(d_{1}+d_{k}\right), \text { for } 2 \leq k \leq K
$$

Part II: Upper bounds. From (12), we have

$$
\begin{aligned}
1-\theta_{K} & =\left(p_{K}-c_{K}\right) \frac{1}{v_{K}-v_{K-1}} \\
& =\left[\left(p_{K}-p_{K-1}\right)+\left(p_{K-1}-c_{K}\right)\right] \frac{1}{v_{K}-v_{K-1}} \\
& >\theta_{K}+\frac{c_{K-1}-c_{K}}{v_{K}-v_{K-1}} \quad\left(\text { since } p_{K-1}>c_{K-1}\right) \\
& =\theta_{K}-d_{K} .
\end{aligned}
$$

Therefore,

$$
\theta_{K}<u_{K} \equiv \frac{1}{2}\left(1+d_{K}\right) .
$$


Similarly, for $1 \leq k \leq K-1$, we have (from (11))

$$
\begin{aligned}
\theta_{k+1}-\theta_{k} & =\left(p_{k}-c_{k}\right)\left(\frac{1}{v_{k+1}-v_{k}}+\frac{1}{v_{k}-v_{k-1}}\right) \\
& >\left(p_{k}-p_{k-1}+p_{k-1}-c_{k}\right) \frac{1}{v_{k}-v_{k-1}} \\
& >\theta_{k}-d_{k},
\end{aligned}
$$

from which the upper bounds of the remaining $\theta_{k}$ 's are established recursively for successively lower $k$ :

$$
\theta_{k}<\frac{1}{2}\left(d_{k}+\theta_{k+1}\right)<u_{k} \equiv \frac{1}{2}\left(d_{k}+u_{k+1}\right),
$$

where $u_{k}$ may be rewritten as

$$
u_{k} \equiv \frac{1}{2^{K-k+1}}+\sum_{i=k}^{K} \frac{1}{2^{i-k+1}} d_{i} .
$$

This completes the proof of the Lemma. Q.E.D.

\section{$7 \quad$ References}

Caplin, Andrew and Barry Nalebuff, "Aggregation and Imperfect Competition: on the Existence of Equilibrium," Econometrica, 59, (1991), 25-59.

Champsaur, Paul and Jean-Charles Rochet, "Multiproduct Duopolists," Econometrica, 57, (1989), 533-557.

De Fraja, Giovanni, "Product Line Competition in Vertically Differentiated Markets," International Journal of Industrial Organization, 14, (1996), 389-414.

Gabszewicz, Jean Jaskold and Jacques-Francois Thisse, "Price Competition, Quality and Income Disparities," Journal of Economic Theory, 20, (1979), 340-359. 
Gabszewicz, Jean Jaskold and Jacques-Francois Thisse, "Entry (and Exit) in a Differentiated Industry," Journal of Economic Theory, 22, (1980), 327338.

Gal-Or, Esther, "Quality and Quantity Competition," Bell Journal of Economics, 14, (1983), 590-600.

Gal-Or, Esther, "Differentiated Industries without Entry Barriers" Journal of Economic Theory, 37, (1985), 310-339.

Itoh, Motoshige, "Monopoly, Product Differentiation and Economic Welfare," Journal of Economic Theory, 31, (1983), 88-104.

Johnson, Justin and David Myatt, "Multiproduct Quality Competition: Fighting Brands and Product Line Pruning," American Economic Review, 93, (2003), 748-774.

Moorthy, Sridhar, "Cournot Competition in a Differentiated Oligopoly," Journal of Economic Theory, 36, (1985), 86-109.

Mussa, Michael and Sherwin Rosen, "Monopoly and Product Quality," Journal of Economic Theory, 18, (1978), 301-317.

Shaked, Avner and John Sutton, "Relaxing Price Competition through Product Differentiation," Review of Economic Studies, 49, (1982), 3-13.

Shaked, Avner and John Sutton, "Natural Oligopolies," Econometrica, 51, (1983), 1469-1483. 